\title{
ESPOROTRICOSIS CUTÁNEA DISEMINADA CON COMPROMISO ARTICULAR EN UNA MUJER CON DIABETES TIPO 2
}

\author{
Sendy Solorzano ${ }^{1, a}$, Renán Ramirez ${ }^{1, b}$, Miguel M Cabada $^{2, b, c}$, Manuel Montoya $^{1, c}$, Ernesto Cazorla ${ }^{1, d}$

\section{RESUMEN} \\ La esporotricosis diseminada es una presentación infrecuente de esta micosis. Aunque ha sido descrita en sujetos \\ inmunocompetentes, con frecuencia existe compromiso de la inmunidad mediada por células T. Reportamos el caso \\ de una mujer con diabetes tipo 2 no controlada que desarrolló esporotricosis cutánea diseminada. El curso de la \\ enfermedad presentó varias complicaciones que incluyeron hepatitis secundaria a itraconazol y artritis de la rodilla \\ con cultivo positivo para Sporothrix schenckii durante el tratamiento con solución saturada de ioduro de potasio. La \\ discusión del caso incluye aspectos de la patogénesis de la esporotricosis diseminada y el manejo de la infección y sus \\ complicaciones.
}

Palabras clave: Esporotricosis; Artritis; Diabetes mellitus; Itraconazol; Yoduro de potasio (fuente: DeCS BIREME)

\section{DISSEMINATED CUTANEOUS SPOROTRICHOSIS WITH JOINT INVOLVEMENT IN A WOMAN WITH TYPE 2 DIABETES}

\begin{abstract}
Disseminated sporotrichosis is a rare presentation of this mycosis. Although it has been described in immunocompetent individuals, there is often T cell-mediated immune compromise. We report the case of a woman with uncontrolled Type 2 diabetes who developed disseminated cutaneous sporotrichosis. The course of the disease presented several complications including hepatitis secondary to itraconazole and knee arthritis with culture positive for Sporothrix schenckii during treatment with saturated solution of potassium iodide. The case discussion includes aspects of the pathogenesis of disseminated sporotrichosis and management of the infection and its complications.
\end{abstract}

Key words: Sporotrichosis; Arthritis; Diabetes mellitus; Itraconazole; Potassium lodide (source: MeSH NLM).

\section{INTRODUCCIÓN}

La esporotricosis humana es causada por un hongo dimórfico ambiental del género Sporothrix. Se han descrito por lo menos cinco especies del complejo de Sporothrix schenckii capaces de producir infección en humanos (S. schenckii sensu stricto, S. braziliensis, S. globosa, S. mexicana, and S. Iuriei) (1). Las formas clínicas linfocutánea y cutánea fija comprenden más del $95 \%$ de los casos ${ }^{(2)}$. En la mayoría de reportes la forma diseminada con o sin compromiso cutáneo es solo el 2 o $3 \%$ de los casos ${ }^{(2,3)}$. García reportó una serie de 44 casos de esporotricosis diagnosticados en la ciudad del Cusco en Perú durante un periodo de 11 años. Sin embargo, ninguno de ellos correspondió a la forma diseminada ${ }^{(4)}$. Similarmente, ninguno de los 85 casos de esporotricosis reportados por Garcia-Izquierdo et al. en Cajamarca, cumplió con la definición de esporotricosis cutánea diseminada ( $\geq 3$ lesiones en 2 o más zonas anatómicas) ${ }^{(5)}$.

La esporotricosis diseminada con compromiso cutáneo y sin compromiso visceral se presenta en sujetos con un sistema inmune normal o con disfunción leve. La esporotricosis diseminada con compromiso visceral (incluyendo la meningitis) se describe usualmente en sujetos con compromiso severo de la inmunidad celular. La diferenciación entre la diseminación cutánea y la visceral es importante ya que esta tiene implicancias terapéuticas y pronósticas. En este manuscrito

\footnotetext{
Departamento de Medicina, Hospital Regional del Cusco. Cusco, Perú.

Departamento de Medicina Interna, University of Texas Medical Branch. Galveston, Texas, EE. UU.

a Médico especialista en dermatología; ${ }^{b}$ médico especialista en medicina interna; ${ }^{\mathrm{c}}$ médico especialista en enfermedades infecciosas; ${ }^{\mathrm{d}}$ médico especialista en gastroenterología

Recibido: 27-08-14 Aprobado: 11-11-14
}

Citar como: Solorzano S, Ramirez R, Cabada MM, Montoya M, Cazorla E. Esporotricosis cutánea diseminada con compromiso articular en una mujer con diabetes tipo 2. Rev Peru Med Exp Salud Publica. 2015;32(1):187-90. 
reportamos un caso de esporotricosis cutánea diseminada con compromiso articular subsecuente en una paciente diagnosticada con diabetes mellitus tipo 2 no controlada durante su hospitalización, y discutimos asuntos relacionados con la patogénesis y tratamiento de la enfermedad.

\section{REPORTE DEL CASO}

Mujer de 53 años, previamente sana, que ingresa al hospital en la ciudad del Cusco, Perú, con 5 semanas de enfermedad que empieza con una lesión papular no dolorosa en la región frontal que, subsiguientemente, se ulceró y aumentó progresivamente de tamaño. Dos semanas más tarde, empezaron a aparecer lesiones nodulares levemente dolorosas en el párpado izquierdo y en regiones preauriculares bilaterales. En la cuarta semana de enfermedad aparecieron nódulos subcutáneos en los miembros inferiores, el tórax anterior y posterior. Durante la semana previa a la presentación, una de las lesiones preauriculares drenó material purulento. La paciente fue sometida durante el curso de su enfermedad a múltiples tratamientos antibióticos, los que incluyeron dicloxacilina y clindamicina, sin ninguna mejoría de las lesiones. La paciente negó haber tenido fiebre, escalofríos, cefalea, compromiso visual, secreción nasal, disfagia, odinofagia, o dolor cervical. Unas semanas antes del inicio de su enfermedad refirió un viaje a la selva de Quillabamba, pero negó labores de jardinería o heridas punzantes en la cara. La paciente tiene contacto cercano con un gato y dos perros en su casa. El gato no presentaba ninguna lesión cutánea, pero los dos perros tenían varias lesiones ulcerosas en el lomo.

En el examen clínico se encontró una úlcera de aproximadamente $4 \mathrm{~cm}$ de diámetro en la región frontal

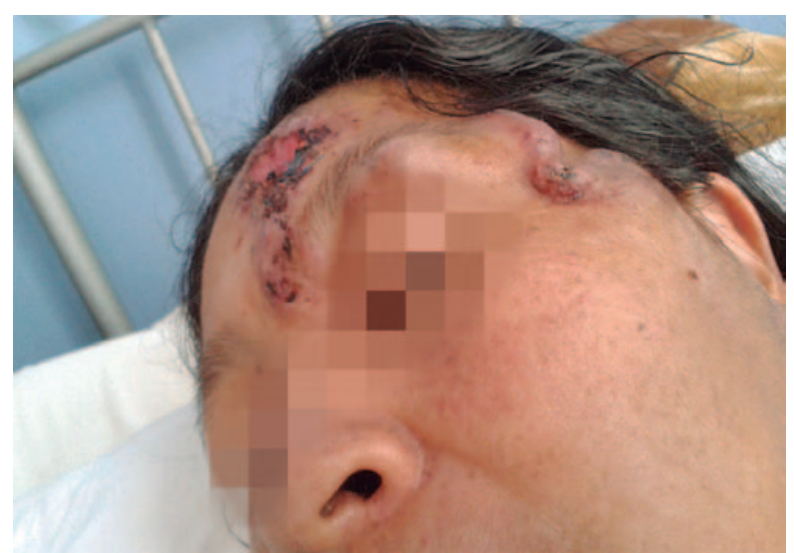

Figura 1. Úlcera facial y múltiples nódulos subcutáneos en la región palpebral y preauricular izquierdas

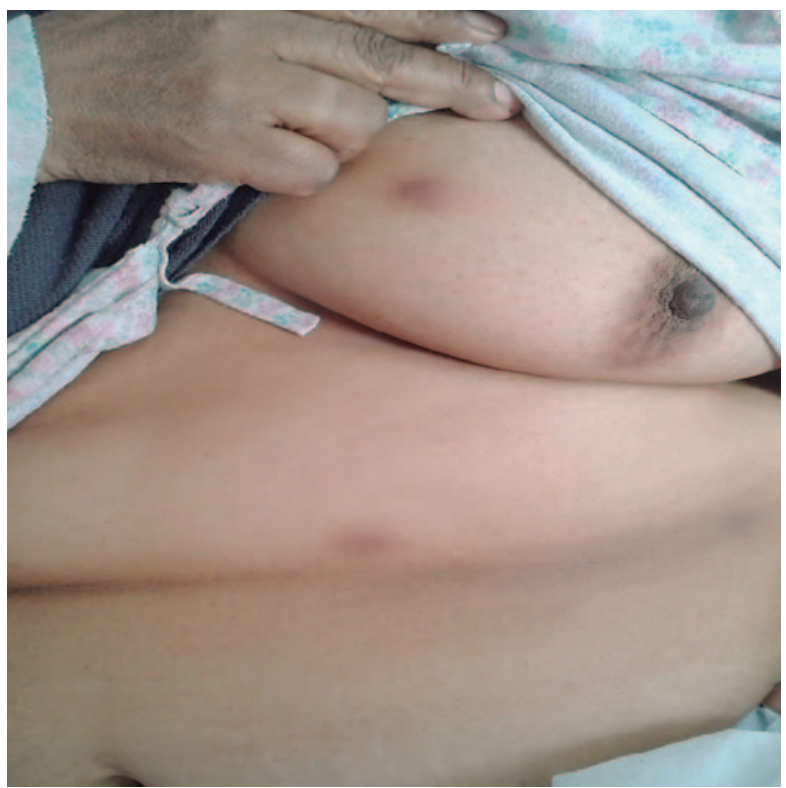

Figura 2. Múltiples nódulos subcutáneos eritematosos en el tórax y en el abdomen

que era levemente dolorosa y tenía una base limpia, la paciente también presentaba lesiones nodulares dolorosas en la región frontal, palpebral izquierda, y preauriculares bilaterales (Figura 1). Los movimiento oculares fueron normales y no se evidenciaron lesiones en las mucosas nasal, oral, o faríngea. Se palparon linfadenopatias cervicales anteriores bilaterales de hasta $2 \mathrm{~cm}$ de diámetro. En la piel se encontraron nueve lesiones nodulares subcutáneas dolorosas de hasta 3 $\mathrm{cm}$ de diámetro en la región torácica anterior y posterior, en el abdomen y en miembros inferiores (Figura 2). El resto del examen físico fue normal. La glucosa sérica en ayunas fue $173 \mathrm{mg} / \mathrm{dL}$. El hemograma completo, nitrógeno ureico, creatinina, y proteínas totales estuvieron dentro de valores normales. Las pruebas de VIH y RPR fueron no reactivas. La radiografía de tórax y la ecografía abdominal fueron reportadas como normales. El frotis de la lesión coloreado con Giemsa fue negativo para leishmaniosis. La coloración hematoxilina-eosina de la biopsia mostró una dermatitis granulomatosa. Las coloraciones Gram y Ziehl Neelsen de la secreción purulenta de la lesión facial fueron negativas. No obstante, el cultivo de la secreción en medio Sabouraud aisló conidióforos y conidios compatibles con Sporothrix schenckii (Figura 3).

La paciente fue diagnosticada con esporotricosis cutánea diseminada y diabetes tipo 2 no controlada. Se inició tratamiento con itraconazol $400 \mathrm{mg}$ en tabletas, diariamente; metformina, y glipizide. Cuatro semanas después de iniciado el tratamiento con itraconazole, el control de enzimas hepáticas mostró 


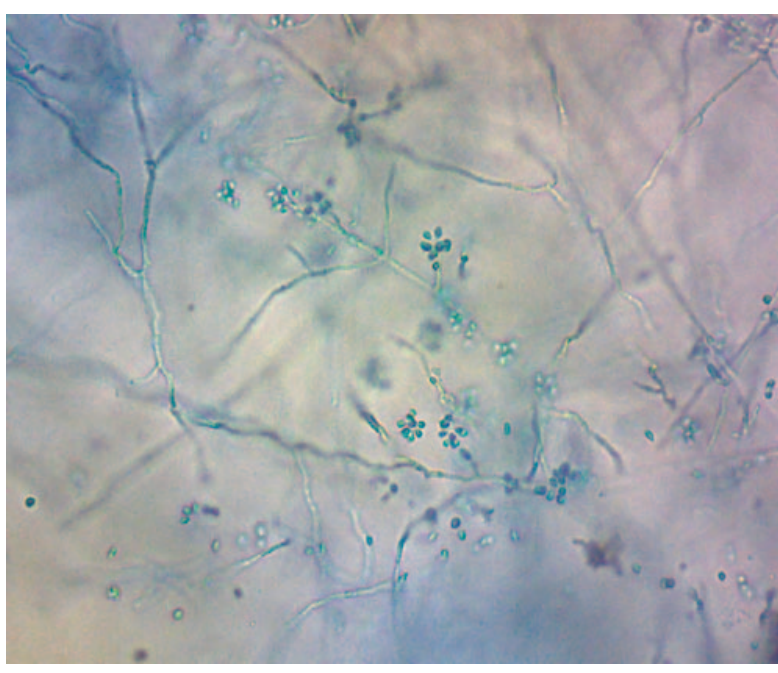

Figura 3. Conidióforos y conidios compatibles con Sporothrix schenckii. Tinción con azul de lactofenol (aumento 40X)

una elevación de diez veces el límite superior normal por lo que el itraconazol fue suspendido. Luego, se inició tratamiento con solución saturada de ioduro de potasio (SSIP) a 1,2 g diarios con resolución gradual de las lesiones cutáneas en el lapso de 2 meses. Sin embargo, durante el tercer mes de tratamiento con SSIP la paciente empezó a presentar aumento de volumen, rubor, y dolor de la rodilla derecha que limitaba la deambulación. Al examen, la paciente presentó tres lesiones maculo-papulares eritematosas en la región malar derecha y artritis de la rodilla derecha. El cultivo de líquido sinovial en medio Sabouraud aisló Sporothrix schenckii. La paciente fue admitida nuevamente al hospital para recibir desoxicolato de anfotericina $B$ endovenoso, seguido de dosis crecientes de itraconazole vía oral alcanzado una dosis de itraconazol de $200 \mathrm{mg}$ diarios sin elevación de las enzimas hepáticas y con resolución completa de las lesiones cutáneas y artritis. La paciente continúo recibiendo itraconazol $200 \mathrm{mg}$ vía oral por un año para el tratamiento de esporotricosis con compromiso articular.

\section{DISCUSIÓN}

La esporotricosis humana se asocia tradicionalmente a exposiciones ocupacionales a plantas y animales. No obstante, la transmisión zoonótica esporádica se ha descrito anteriormente ${ }^{(6)}$. Además, también se han reportado brotes importantes de esporotricosis transmitida por gatos (7). En nuestra paciente, la transmisión pudo estar asociada a la exposición a gatos o perros, lo que ha sido reportado como un factor de riesgo para la transmisión esporádica en el Perú (8). En nuestra paciente no se pudo identificar ninguna otra exposición de riesgo para esporotricosis.

La esporotricosis diseminada es poco común y su patogénesis no es entendida completamente. Es posible que el tipo de exposición, la susceptibilidad individual, y los factores de virulencia del hongo cumplan una función en la diseminación. En un brote de esporotricosis zoonótica en Brasil, el inóculo y la intensidad de exposición a gatos infectados se reportaron como posibles explicaciones para una incidencia inusualmente alta de esporotricosis cutánea diseminada (16-18\%) ${ }^{(9)}$. En la mayoría de casos de diseminación se reportan alteraciones de la respuesta inmune mediada por células $T$ y el grado de alteración inmunitaria se relaciona con la severidad de la presentación (2). La diabetes tipo 2 y el alcoholismo acompañan a la esporotricosis cutánea diseminada con frecuencia, mientras que el VIH/SIDA se asocia con frecuencia a enfermedad diseminada con compromiso visceral y funguemia ${ }^{(7,10)}$. La glicoproteina Gp70 es una adesina importante en la superficie de Sporothrix spp. Un estudio reciente sugiere una expresión diferenciada de Gp70 entre especies y dentro de las mismas especies que causan presentaciones clínicas distintas ${ }^{(1)}$. En nuestra paciente, la diabetes tipo 2 no controlada pudo haber sido un factor contribuyente para la diseminación de esporotricosis. Ninguna otra causa de inmunosupresión fue aparente en esta paciente.

Mientras que el itraconazol es la medicina recomendada para el tratamiento de esporotricosis linfática y cutánea, la anfotericina B es la droga recomendada para la enfermedad visceral ${ }^{(11)}$. La confusión sobre la elección del tratamiento para individuos con enfermedad diseminada probablemente surge de la sobreposición de las definiciones. A pesar de llamarse esporotricosis "cutánea diseminada", esta forma de la enfermedad tiene una respuesta excelente al itraconazol oral. La serie más grande de casos de esporotricosis tratada con itraconazol se originó en un brote de transmisión zoonótica en Brasil que comprometió a 645 sujetos. Cuarenta y tres sujetos en la serie presentaron enfermedad cutánea diseminada. Treinta y siete ( $86 \%$ ) de estos sujetos curaron después del tratamiento con itraconazol $100 \mathrm{mg}$ vía oral por una media de 12 semanas ${ }^{(12)}$.

La paciente recibió, inicialmente, una alta dosis de itraconazol con mejoría clínica, pero con efectos tóxicos en el hígado que llevaron a la suspensión del tratamiento. Sin embargo, el tratamiento con itraconazol a dosis habituales parece ser efectivo y seguro en pacientes con esporotricosis diseminada con compromiso cutáneo y sin compromiso visceral. En contraste, la SSIP no se recomienda para la enfermedad diseminada debido a su menor efectividad en comparación con itraconazol. 
El compromiso articular que se desarrolló durante el tratamiento con SSIP requiere de dosis altas de itraconazol por al menos 12 meses ${ }^{11}$. Tratamientos más cortos pueden resultar en recurrencia de la enfermedad ${ }^{13,14}$. En nuestra paciente, el tratamiento con itraconazol $400 \mathrm{mg}$ diarios causó hepatitis severa. Por eso, se decidió administrar el tratamiento de segunda línea que consiste en anfotericina $B$ seguida de dosis crecientes de itraconazol con vigilancia cercana de las enzimas hepáticas.
Contribuciones de autoría: SS, RR, MMC, MMC y EC intervinieron en el cuidado de la paciente, recolectaron la información, revisaron críticamente el borrador del manuscrito, y aprobaron su versión final. Además, MMC redactó el manuscrito y sus distintas versiones.

Fuentes de financiamiento: autofinanciado.

Conflictos de interés: los autores no tiene conflictos de interés que declarar.

\section{REFERENCIAS BIBLIOGRÁFICAS}

1. Castro RA, Kubitschek-Barreira PH, Teixeira PA, Sanches GF, Teixeira MM, Quintella LP, et al. Differences in cell morphometry, cell wall topography and Gp70 expression correlate with the virulence of Sporothrix brasiliensis clinical isolates. PLoS One. 2013;8(10):e75656.

2. Vásquez-del-Mercado E, Arenas R, Padilla-Desgarenes C. Sporotrichosis. Clin Dermatol. 2012 JulAug;30(4):437-43. doi: 10.1016/j. clindermatol.2011.09.017.

3. Song Y, Li SS, Zhong SX, Liu YY, Yao L, Huo SS. Report of 457 sporotrichosis cases from Jilin province, northeast China, a serious endemic region. J Eur Acad Dermatol Venereol. 2013 Mar;27(3):313-8. doi: 10.1111/j.14683083.2011.04389.x.

4. Garcia R. Esporotricosis en la altura del Cusco - Perú. Nueva zona endémica -Experiencia de once años. Folia Dermatol Peru. 1998;9(1):1-5

5. García-Izquierdo $M$, Urquiaga $T$, López N. Esporotricosis cutanea en el Hospital Regional de Cajamarca. Rev Peru Dermatol. 2001;11(2):87-91.

6. Rees RK, Swartzberg JE. Felinetransmitted sporotrichosis: A case study from California. Dermatol Online J. 2011 Jun 15;17(6):2.
7. Freitas DF, do Valle AC, de Almeida Paes R, Bastos FI, Galhardo MC. Zoonotic sporotrichosis in Rio de Janeiro, Brazil: a protracted epidemic yet to be curbed. Clin Infect Dis. 2010 Feb 1;50(3):453. doi: 10.1086/649891.

8. Lyon GM, Zurita S, Casquero J, Holgado W, Guevara J, Brandt ME, et al. Population-based surveillance and a case-control study of risk factors for endemic lymphocutaneous sporotrichosis in Peru. Clin Infect Dis. 2003 Jan 1;36(1):34-9.

9. De Lima Barros MB, de Oliveira Schubach A, Galhardo MC, Schubach TM, dos Reis RS, Conceição MJ, et al. Sporotrichosis with widespread cutaneous lesions: report of 24 cases related to transmission by domestic cats in Rio de Janeiro, Brazil. Int J Dermatol. 2003 Sep;42(9):677-81.

10. Freitas DF, de Siqueira Hoagland B, do Valle AC, Fraga BB, de Barros MB, de Oliveira Schubach A, et al. Sporotrichosis in HIV-infected patients: report of 21 cases of endemic sporotrichosis in Rio de Janeiro, Brazil. Med Mycol. 2012 Feb;50(2):170-8. doi: $10.3109 / 13693786.2011 .596288$.

11. Kauffman CA, Bustamante B, Chapman SW, Pappas PG. Clinical practice guidelines for the management of sporotrichosis: 2007 update by the Infectious Diseases Society of America. Clin Infect Dis. 2007 Nov $15 ; 45(10): 1255-65$.

12. De Lima Barros MB, Schubach AO, de Vasconcellos Carvalhaes de Oliveira R, Martins EB, Teixeira JL, Wanke B. Treatment of cutaneous sporotrichosis with itraconazole--tudy of 645 patients. Clin Infect Dis. 2011 Jun 15;52(12):e200-6. doi: $10.1093 / \mathrm{cid} /$ cir245.

13. Appenzeller S, Amaral TN, Amstalden EMI, Bertolo MB, Neto JFM, Samara AM, et al. Sporothrix schenckii infection presented as monoarthritis: report of two cases and review of the literature. Clin Rheumatol. 2006 Nov;25(6):926-8.

14. Ramos-e-Silva M, Vascocelos C, Carneiro S, Cestari T. Sporotrichosis. Clin Dermatol. 2007 MarApr;25(2):181-7.

Correspondencia: Miguel M Cabada Dirección: Cal. José Carlos Mariátegui J-6, Urbanización Sata Mónica, Wanchaq,

Cusco, Perú.

Teléfono: (+51) 964506444

Correo electrónico:micabada@utmb.edu 